\title{
INVOLVING SYMMETRIES OF RIEMANN SURFACES TO A STUDY OF THE MAPPING CLASS GROUP
}

\author{
Grzegorz Gromadzki and Micha£ Stukow
}

\begin{abstract}
A pair of symmetries $(\sigma, \tau)$ of a Riemann surface $X$ is said to be perfect if their product belongs to the derived subgroup of the group $\mathrm{Aut}^{+}(X)$ of orientation preserving automorphisms. We show that given $g \neq 2,3,5,7$ there exists a Riemann surface $X$ of genus $g$ admitting a perfect pair of symmetries of certain topological type. On the other hand we show that a twist can be written as a product of two symmetries of the same type which leads to a decomposition of a twist as a product of two commutators: one from $\mathcal{M}^{\prime}$ which entirely lives on a Riemann surface and one from $\mathcal{M}^{ \pm \prime}$. As a result we obtain the perfectness of the mapping class group $\mathcal{M}_{g}$ for such $g$ relying only on results of Birman [1] but not on influential paper of Powell [6] nor on Johnson's rediscovery of Dehn lantern relation [3] and nor on recent results of Korkmaz-Ozbagci [4] who found explicit presentation of a twist as a product of two commutators.
\end{abstract}

Let $X$ be a compact surface of genus $g \geq 2$ and let $\mathcal{M}^{ \pm}=\mathcal{M}_{g}^{ \pm}$be the extended mapping class group which is the group of isotopy classes of homeomorphisms of $X$. The mapping class group $\mathcal{M}$ is the subgroup of $\mathcal{M}^{ \pm}$consisting of the isotopy classes of orientation preserving homeomorphisms. It is well known [5] that $\mathcal{M}$ is generated by $3 g-1$ canonical twists and together with a symmetry (by which we mean an orientation reversing involution) they generate $\mathcal{M}^{ \pm}$. Classical results of Harnack and Weichhold assert that the conjugacy class of a symmetry $\sigma$ in $\mathcal{M}^{ \pm}$is determined by its topological type $\varepsilon k$, where $k$ is the number of connected components of the set $\operatorname{Fix}(\sigma)$ of fixed points of $\sigma$ (ovals in Hilbert's terminology) and $\varepsilon=\varepsilon(\sigma)$ is the separability index, which is equal to -1 or +1 according to whether $X \backslash \operatorname{Fix}(\sigma)$ is connected or

2000 Mathematics Subject Classification. Primary: 57A05, 30F10; Secondary: 57M50, 20F05.

Key words. Mapping class group, symmetries of Riemann surfaces.

Both authors supported by BW5100-5-0080-3. 
not. Let $\sigma$ be a symmetry with $k$ ovals $c_{1}, \ldots, c_{k}$ and let $h_{c_{i}}$ be a twist about $c_{i}$. Then $\sigma h_{c_{i}}$ belongs to the class of a non-separating symmetry with $k-1$ ovals $c_{1}, \ldots, \hat{c}_{i}, \ldots, c_{k}$. Iterating this construction for a separating symmetry with $g+1$ ovals we obtain

Theorem 1. Given $g \geq 1$ and $k$ in range $0 \leq k \leq g-1$ there is a pair of non-separating symmetries on a surface of genus $g$ with $k$ and $k+1$ ovals whose product is a twist. In particular the extended mapping class group is generated by involutions.

A pair of symmetries of a Riemann surface $X$ is said to be perfect if their product belong to the derived subgroup of the group of automorphisms of $X$.

Lemma 2. Given an even integer $g \geq 4$ there exists a Riemann surface $X$ of genus $g$ admitting a perfect pair of non-separating symmetries with 1 and 2 ovals. For a given odd integer $g \geq 9$, there exists a Riemann surface of genus $g$ admitting a perfect pair of non-separating symmetries with 2 and 3 ovals.

Proof: We shall prove the lemma using theory of Fuchsian and NEC groups; we send the reader to [2] where he can find necessary background. Let $\Lambda$ be an NEC group with signature $(0 ;+;[-] ;\{(2, . s ., 2,4)\})$, where $s=(g+4) / 2$ and let $G=\mathrm{D}_{4} \oplus \mathrm{Z}_{2}=\left\langle a, b \mid a^{2}, b^{2},(a b)^{4}\right\rangle \oplus\left\langle x \mid x^{2}\right\rangle$. Let $\theta: \Lambda \rightarrow G$ be an epimorphism induced by the assignment: $\theta(e)=1$, $\theta\left(c_{0}\right)=\theta\left(c_{s+1}\right)=a, \theta\left(c_{1}\right)=x, \theta\left(c_{2}\right)=b(a b)^{2(s+1)}, \theta\left(c_{3}\right)=x(a b)^{2}$ and $\theta\left(c_{i}\right)=b(a b)^{2(i+s)}$. Neither a reflection nor an elliptic elements belong $\Gamma=\operatorname{ker} \theta$ and so it is a surface Fuchsian group. Thus $X=\mathcal{H} / \Gamma$ is a Riemann surface on which $G$ acts as a group of automorphisms. By the Hurwitz Riemann formula $X$ has genus $g$. Now we shall show that $\sigma=x$ is a non-separating symmetry of $X$ with 1 oval. For, observe first that $\sigma$ is central in $G$ and consider induced epimorphism $\tilde{\theta}: \Lambda \rightarrow G /\langle\sigma\rangle$. Then $\Gamma_{\sigma}=\operatorname{ker} \tilde{\theta}$ is a surface NEC group which by $[\mathbf{2}, 2.3 .3]$ has 1 empty period-cycle. In addition by $[2,2.1 .3] \Gamma_{\sigma}$ has the $\operatorname{sign}-$ and so $\sigma$ is a non-separating symmetry with 1 oval indeed. In the same way one can show that $\tau=x(a b)^{2}$ is a non-separating symmetry with 2 ovals. Finally, $\sigma \tau=[a b, a \sigma]$ belongs to the derived group of $\mathrm{Aut}^{+}(X)$.

The case of odd $g$ is similar. As before $G=\mathrm{D}_{4} \oplus \mathrm{Z}_{2}$ but now let $\Lambda$ be an NEC group with signature $(0 ;+;[-] ;\{(2, . s ., 2,4,4)\})$, where $s=$ $(g+1) / 2$. We define an epimorphism $\theta: \Lambda \rightarrow G$ by the assignment: $\theta(e)=1, \theta\left(c_{0}\right)=\theta\left(c_{s+2}\right)=a, \theta\left(c_{1}\right)=\theta\left(c_{4}\right)=x, \theta\left(c_{2}\right)=\theta\left(c_{s+1}\right)=b$, $\theta\left(c_{3}\right)=(a b)^{2} x$ and $\theta\left(c_{i}\right)=(a b)^{2(i+s)} a$ for the remaining reflections $c_{i}$. 
Now $X$ is a Riemann surface of genus $g$ and $\tau=x(a b)^{2}, \sigma=x$ represent two non-separating symmetries of $X$ with 2 and 3 ovals respectively.

Corollary 3. The mapping class group $\mathcal{M}_{g}$ of a compact Riemann surface of genus $g \neq 2,3,5,7$ is perfect.

Proof: Take $k=1$ or 2 if $g$ is even or odd respectively. By Theorem 1 there is a pair $\left(\sigma^{\prime}, \tau^{\prime}\right)$ of non-separating symmetries with $k$ and $k+1$ ovals whose product $\sigma^{\prime} \tau^{\prime}$ is a twist and by Lemma 2 there is a perfect pair $(\sigma, \tau)$ of such symmetries. By the mentioned above theorem of Weichhold, $\sigma^{\prime}=\alpha \sigma \alpha^{-1}$, and $\tau^{\prime}=\beta \tau \beta^{-1}$ for some $\alpha, \beta \in \mathcal{M}$. So $h=\alpha^{-1} \sigma^{\prime} \tau^{\prime} \alpha=$ $\sigma \tau\left[\tau, \alpha^{-1} \beta\right]$ is a twist and thus $\mathcal{M} \subseteq \mathcal{M}^{ \pm \prime}$. Now $\mathcal{M}^{ \pm} / \mathcal{M}=\mathrm{Z}_{2}$ and by $[\mathbf{1}],\left|\mathcal{M} / \mathcal{M}^{\prime}\right| \leq 2$. So $\mathcal{M}^{ \pm} / \mathcal{M}^{\prime}$ is abelian as a group of order $\leq 4$ and therefore $\mathcal{M}^{ \pm \prime} \subseteq \mathcal{M}^{\prime}$.

Remark. Observe that the proof of the second part of Lemma 2 works only for $s \geq 5$ which forces $g \geq 9$. We guess that probably also for $g=3,5,7$, Riemann surfaces which admits perfect pairs of non-separating symmetries with $k$ and $k+1$ ovals exists. However our aim was rather to show that the extended mapping class group is generated by classes of symmetries and to show how one can use symmetries surfaces to the study of the mapping class involving methods of Riemann surfaces than in the proof of its perfectness for itself and so we have skipped this question.

\section{References}

[1] J. S. BIRMAn, Abelian quotients of the mapping class group of a 2-manifold, Bull. Amer. Math. Soc. 76 (1970), 147-150.

[2] E. Bujalance, J. J. Etayo, J. M. Gamboa and G. Gromadzki, "Automorphism groups of compact bordered Klein surfaces. A combinatorial approach", Lecture Notes in Mathematics 1439, SpringerVerlag, Berlin, 1990.

[3] D. L. Johnson, Homeomorphisms of a surface which act trivially on homology, Proc. Amer. Math. Soc. 75(1) (1979), 119-125.

[4] M. Korkmaz and B. Ozbagci, Minimal number of singular fibers in a Lefschetz fibration, Proc. Amer. Math. Soc. 129(5) (2001), $1545-1549$

[5] W. B. R. Lickorish, A finite set of generators for the homeotopy group of a 2-manifold, Proc. Cambridge Philos. Soc. 60 (1964), 769-778.

[6] J. Powell, Two theorems on the mapping class group of a surface, Proc. Amer. Math. Soc. 68(3) (1978), 347-350. 
Institute of Mathematics

University of Gdańsk

Wita Stwosza 57

80-952 Gdańsk

Poland

E-mail address: greggrom@math.univ.gda.pl

E-mail address: trojkat@math.univ.gda.pl

Rebut el 24 de febrer de 2003. 